\title{
Leptin deficiency affects glucose homeostasis and results in adiposity in zebrafish
}

\author{
Junling He1,2,*, Yi Ding2,*, Natalia Nowik ${ }^{(2,3}$, Charel Jager1, Muhamed N H Eeza4, A Alia(D4,5, Hans J Baelde ${ }^{1} 1$ and \\ Herman P Spaink²
}

\begin{abstract}
1Department of Pathology, Leiden University Medical Center, Leiden, The Netherlands 2Department of Animal Sciences and Health, Institute of Biology, Leiden University, Leiden, The Netherlands ${ }^{3}$ Department of Animal Anatomy, Faculty of Veterinary Medicine, University of Warmia and Mazury in Olsztyn, Olsztyn, Poland 4 Institute of Medical Physics and Biophysics, University of Leipzig, Leipzig, Germany

5Leiden Institute of Chemistry, Leiden University, Leiden, The Netherlands
\end{abstract}

Correspondence should be addressed to H P Spaink: h.p.spaink@biology.leidenuniv.nl

*(J He and Y Ding contributed equally to this work)

\begin{abstract}
Leptin is a hormone which functions in the regulation of energy homeostasis via suppression of appetite. In zebrafish, there are two paralogous genes encoding leptin, called lepa and lepb. In a gene expression study, we found that the lepb gene, not the lepa gene, was significantly downregulated under the state of insulin-resistance in zebrafish larvae, suggesting that the lepb plays a role in glucose homeostasis. In the current study, we characterised lepb-deficient $\left(l e p b^{-/-}\right)$adult zebrafish generated via a CRISPR-CAS9 gene editing approach by investigating whether the disruption of the lepb gene would result in the development of type 2 diabetes mellitus (T2DM) and diabetic complications. We observed that lep $b^{-/-}$adult zebrafish had an increase in body weight, length and visceral fat accumulation, compared to age-matched control zebrafish. In addition, lep $\mathrm{b}^{-/-}$zebrafish had significantly higher blood glucose levels compared to control zebrafish. These data collectively indicate that lep $b^{-1-}$ adult zebrafish display the features of T2DM. Furthermore, we showed that lept-l- adult zebrafish had glomerular hypertrophy and thickening of the glomerular basement membrane, compared to control zebrafish, suggesting that lepb-l- adult zebrafish develop early signs of diabetic nephropathy. In conclusion, our results demonstrate that lepb regulates glucose homeostasis and adiposity in zebrafish, and suggest that lep $b^{-/-}$mutant zebrafish are a promising model to investigate the role of leptin in the development of T2DM and are an attractive model to perform mechanistic and therapeutic research in T2DM and its complications.
\end{abstract}

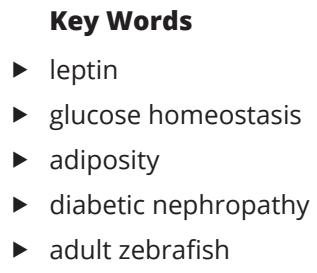

Journal of Endocrinology (2021) 249, 125-134

\section{Introduction}

Human leptin is a $16-\mathrm{kDa}$ protein hormone which is predominantly secreted by adipocytes (Zhang et al. 1994). Leptin plays a role in diverse physiological processes including energy homeostasis (Ahima et al. 1998), immune regulation (Lord et al. 1998, Matarese et al. 2005), endocrine regulation (Meier \& Gressner 2004), and reproduction (Caprio et al. 2001, Licinio et al. 2004). Congenital leptin deficiency causes extreme obesity 
in children (Farooqi et al. 1999, Funcke et al. 2014). Rodents lacking the gene encoding leptin are commonly characterised by hyperphagia, obesity, insulin resistance and impaired glucose tolerance. For instance, leptindeficient mice ( $o b / o b$ mice) exhibit the features of obesity and type 2 diabetic mellitus (T2DM) (Drel et al. 2006). Growing evidence suggests that leptin treatment has a beneficial effect on glucose metabolism (Yu et al. 2008, Fujikawa et al. 2010, Hedbacker et al. 2010, German et al. 2011) and insulin resistance (Muzzin et al. 1996, Pocai et al. 2005, Park et al. 2008, German et al. 2009), indicating that leptin might be a crucial factor in the development of T2DM. However, the function of leptin in the pathogenesis of T2DM is still obscure.

Leptin and leptin receptor are highly conserved across mammalian species. The most widely used animal models in T2DM research are the congenial leptin- or leptin receptor-deficient rodent models, such as $o b / o b$ and $d b / d b$ mice. Besides, the basic structural features and intracellular signaling mechanisms of leptin and its receptor appear to be conserved throughout vertebrates (Denver et al. 2011). Several studies have reported that administration of exogenous leptin in fish reduces food intake, indicating the conservation of the function of the leptin signaling system throughout vertebrates (Volkoff et al. 2003, Murashita et al. 2008, Aguilar et al. 2011). In recent decades, zebrafish have become a promising animal model with numerous advantages, including fast development and generation time, small size, easily accessible larvae, and cost-effective breeding. In zebrafish, there are two divergent leptin paralogous: lepa and lepb, and one leptin receptor gene (lepr) (Gorissen et al. 2009). Michel et al. (2016) demonstrated that lepr-deficient zebrafish larvae have increased numbers of $\beta$-cells and increased insulin mRNA expression, compared to control zebrafish larvae. They also found that the leptin receptor deficiency contributes to higher blood glucose levels in adult zebrafish. Their findings indicated that the regulation of glucose homeostasis by the leptin receptor is conserved across vertebrates. However, they observed no significant difference in the whole body adiposity phenotype between lepr-deficient zebrafish and control zebrafish at the adult stage. On the other hand, Chisada et al. (2014) showed that the deletion of leptin receptor in medaka results in a modest increase in visceral fat accumulation compared to the WT medaka fish. More recently, Audira et al. (2018) found that lepa-deficient adult zebrafish display an obesity phenotype. Thus, there are some conflicting results in the literature regarding the relationship between disruption of leptin signaling and adiposity in zebrafish.
In a previous study, our group found that the lepb gene, not the lepa gene, was significantly downregulated in zebrafish larvae under an insulin-resistance state, resulting from acute hyperinsulinemia, which suggests that $l e p b$ plays a role in insulin homeostasis in zebrafish (Marin-Juez et al. 2014). Since the roles of lepa and lepr in zebrafish have already been investigated by other groups, therefore, the focus in the current work is to study the function of the lepb gene in zebrafish at an adult stage to obtain more insights into the relationship between the leptin signaling pathway and T2DM.

This study aims to investigate whether lepb deficiency contributes to the development of T2DM in adult zebrafish. To address this question, we examined the body weight and length, blood glucose levels, and the body fat distribution in 1.5 years old lepb ${ }^{-/-}$adult zebrafish and compared them to age-matched WT controls. Furthermore, we examined the renal histopathologic changes of these zebrafish by performing hematoxylin and eosin (HE) or Periodic-acid Schiff (PAS) staining, and transmission electron microscopy (TEM) methods.

\section{Methods and materials}

\section{Animals}

Zebrafish strains were handled in compliance with the local animal welfare regulations and maintained according to standard protocols (zfin.org). The use of adult zebrafish was approved by the local animal welfare committee (DEC) of the University of Leiden (license number: AVD1060020171767) and adhered to the international guidelines specified by the EU Animal Protection Directive 2010/63/EU.

The CRISPR-CAS9 gene editing approach was used to knock out the lepb in the ABTL zebrafish to generate the lepb-deficient zebrafish. The sgRNA CTACCCAATCCCGAGACCCC targeted the exon 2 in lepb. The lepb primer (for: $5^{\prime}-A G G A A C T G G C C G T C T C A C A G-3^{\prime}$; rev: $5^{\prime}$-CGGGGAAGGCTGTTTCTTCTT-3') was used for lepb-deficient zebrafish genotyping. Sanger sequencing showed that a $7 \mathrm{bp}\left(l e p b^{7-17-}\right)$ or $8 \mathrm{bp}\left(l e p b^{8-/ 8-}\right)$ stretch of nucleotides was missing in the lepb gene in two different mutant lines (Supplementary Fig. 1A, see section on supplementary materials given at the end of this article). Both deletions resulted in a frameshift mutation of lepb (Supplementary Fig. 1B). The deletion of the lepb gene was in codon 110 in zebrafish, which is close to the non-sense mutation in codon 105 of the leptin gene of C57BL/6J $o b / o b$ mice. In the C57BL/6J ob/ob mice, this mutation in 
the leptin gene leads to a truncated protein that cannot bind to the leptin receptor anymore (Zhang et al. 1994). Therefore, although we cannot be sure that our mutant has a complete null phenotype, it is very likely that our truncation in the lepb gene leads to a leptin $\mathrm{b}$ protein which cannot bind to the leptin receptor in the zebrafish.

The $l e p b^{+/+}$and lepb $b^{-/-}$zebrafish are obtained from an incross of lepb heterozygous fish (lep ${ }^{+-}$); (Supplementary Fig. 1C). $L e p b^{+/+}$, lep $b^{+/-}$and lep $b^{-/-}$zebrafish were mixed and raised in the same tank before genotyping. After genotyping, $l e p b^{+/+}$, lep $b^{+/-}$and lep $b^{-/-}$fish were separated and raised in different sizes of tanks depending on the number of fish per volume of water. The ABTL (control) fish of 1.5 years old were kept in the big-sized-tanks. We raised all zebrafish in the same circulating water system and maintained the normal mixed-sex environment. Adult zebrafish were fed twice per day. One meal is a mixture of GEMMA Micro 300 and GEMMA Diamond (Skretting; Nutreco company) whose amount is based on 5\% of zebrafish body weight; another is the life Artemia (ZebCare). Both amounts are positively proportional to the number of fish per tank and given by highly skilled caretakers.

We included both $l e p b^{7-/ 7-}$ and lepb $b^{8-/ 8-}$ mutants in the current study since two independent mutants have more certainty to rule out off-target effects of CRISPR-CAS9 approach. The age-matched ABTL adult zebrafish of the same lineage as used for the CRISPR-CAS9 procedure were used as the controls. In an independent study, a WT $\left(l e p b^{+/+}\right)$zebrafish line, resulting from an incross of heterozygotes lep $b^{7+/}$ was compared with the ABTL control, showing no significant difference in blood glucose levels (Supplementary Fig. 2; female: $t_{(3)}=-0.149$, $P=0.891$; male: $\left.t_{(3)}=-2.820, P=0.067\right)$, further excluding off-target effects of the CRISPR-CAS9 procedure. For the current study, adult zebrafish (ABTL female fish, $n=9$; lep $b^{-1-}$ female fish, $n=11$; ABTL male fish, $n=9$; lepb $b^{-/}$ male fish, $n=15$ ) were sacrificed at the age of 1.5 years, since we observed that all lep $b^{-/-}$mutants displayed an obese phenotype, compared to control zebrafish. Because there was no difference in body weight, body length, body mass index (BMI), and $2 \mathrm{~h}$ postprandial blood glucose levels between $l e p b^{7-17-}$, lep $b^{8-/ 8-}$ and $l e p b^{7-/ 8-}$ mutants (Supplementary Fig. 3), we pooled lepb $b^{7 / 7-}$, lep $b^{8-/ 8-}$ and $l e p b^{7-18-}$ mutants into one group which is named as lep $b^{-/-}$ for the subsequent analysis.

\section{Body weight and body length measurement}

Two hours after feeding, fish were sacrificed by putting them into ice-cold aquarium water which was filled with ice chips to maintain the temperature near $0^{\circ} \mathrm{C}$ for $3-6 \mathrm{~s}$. After there was no response of the fish to external stimuli, the euthanized fish was placed on a paper tissue, drying the body as much as possible. Then, the fish size (from the tip of the mouth to the caudal peduncle) was measured with a calliper, and the fish were weighed with a precision analytical scale.

\section{Blood collection}

A steel blade was used to cut the fish between anal fin and caudal fin just after euthanasia to collect zebrafish blood. The blood was collected into a $500 \mu \mathrm{L}$ Eppendorf tube with a $20 \mu \mathrm{L}$ micro-pipet. Following the blood collection, the remaining body of the zebrafish was fixed in $4 \%$ paraformaldehyde (PFA). After $2 \mathrm{~h}$ of coagulation at room temperature, the blood was spun down in a centrifuge at $13000 \boldsymbol{g}$ for $10 \mathrm{~min}$. Subsequently, the blood serum and the blood cells were separated into different Eppendorf tubes and kept at $-80^{\circ} \mathrm{C}$ for the further analysis.

\section{Blood glucose level measurement}

A PicoProbe ${ }^{\mathrm{TM}}$ Glucose Fluorometric Assay kit (Biovision, Milpitas, CA) was used to measure the glucose levels in the serum of zebrafish. The serum samples were diluted $50 \times$ with milli-Q water. Nine microliters of the diluted serum was added into a 96-well white plate, and then the total volume was adjusted to $50 \mu \mathrm{L}$ with $36 \mu \mathrm{L}$ glucose assay buffer and $5 \mu \mathrm{L}$ reaction mix which was composed of $0.5 \mu \mathrm{L}$ PicoProbe ${ }^{\mathrm{TM}}, 1 \mu \mathrm{L}$ glucose substrate mix, $1 \mu \mathrm{L}$ glucose enzyme mix and $2.5 \mu \mathrm{L}$ glucose assay buffer. The $100 \mathrm{mM}$ glucose standard was diluted into a series of wells in 96-well plates to generate $0,1.5,3,6,9,12$, $15 \mu \mathrm{M} /$ well for making the glucose standard curve. The volumes of different concentrations of glucose standards were also adjusted to $50 \mu \mathrm{L}$ with glucose assay buffer and reaction mix. The samples were incubated for $30 \mathrm{~min}$ at $37^{\circ} \mathrm{C}$, protected from light. Fluorescence was measured at excitation/emission $=535 / 587 \mathrm{~nm}$ in a microtiter plate reader. The concentrations of glucose in the serum of zebrafish were calculated according to the glucose standard curve.

\section{MRI measurement}

After one week fixation in $4 \%$ PFA at $4^{\circ} \mathrm{C}$, the whole adult fish were washed twice with PBS and then transferred to MR silent liquid (Fomblin, perfluoropolyether) for MRI measurement. All MRI scans were performed at 
$300 \mathrm{MHz}$ Bruker vertical wide-bore system, using a birdcage radiofrequency coil with an inner diameter of $10 \mathrm{~mm}$. Data acquisition and processing were performed with Para Vision 5.1 (Bruker Biospin, Germany). Before each measurement, the magnetic field homogeneity was optimized by shimming. Each session of measurements began with a multi-slice orthogonal gradient-echo sequence for position determination and selection of the desired region for subsequent experiments.

For anatomical images, a rapid acquisition with relaxation enhancement (RARE) sequences was used. Basic measurement parameters used for the RARE sequence were: echo time $(\mathrm{TE})=8.5 \mathrm{~ms}$ with an effective echo time of $18.1 \mathrm{~ms}$; repetition time $(\mathrm{TR})=3000 \mathrm{~ms}$; number of scans $(\mathrm{ns})=12$; total $\mathrm{scan}$ time $=17 \mathrm{~min}$; RARE factor $=4$. The field of view (FOV) was $1.2 \mathrm{~cm}$ with a matrix size of $256 \times 256$, the slice thickness was $0.2 \mathrm{~mm}$, and the interslice distance was also $0.2 \mathrm{~mm}$.

For selective fat imaging, a Chemical Shift Selective (CHESS) sequence was used. The CHESS consists of a single frequency-selective excitation pulse with a flip angle of $\pi / 2$ followed by a dephasing gradient (homogeneity spoiling gradient). The procedure leaves the spin system in a state where no net magnetization of the unwanted component is retained while the wanted component remains entirely unaffected in the form of z-magnetization. A narrow bandwidth of $90^{\circ}$ Gaussian pulse was used for on resonance frequency selective excitation. Further basic parameters used are as follows: $\mathrm{TE}=13.3 \mathrm{~ms}$; $\mathrm{TR}=800 \mathrm{~ms}$; ns = 16; Scan time 27 min.

For quantitative analysis of fat in CHESS images, the image slices were exported and analyzed in Image J software (https://imagej.nih.gov/ij/). By using a plugin, the area of the fish was defined, and a certain threshold was adjusted to eliminate any contribution of noise. Subsequently, the hyperintense signal of fat was calculated. The data were exported to Origin Pro v. 8 software for further analysis.

\section{Renal histopathology}

After 1 week fixation in $4 \%$ PFA at $4^{\circ} \mathrm{C}$, the whole adult zebrafish were washed twice with PBS and then transferred to EDTA (100 mM, pH 8) for the decalcification at room temperature for another 1 week, followed by embedding

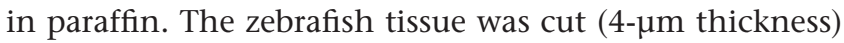
on a Leica microtome (Wetzlar) and then stained with HE or PAS using standard protocols.

Stained slides were digitalized using a Philips UltraFast Scanner 1.6 RA (Philips Electronics, Amsterdam, the Netherlands). The surface areas $\left(\mu \mathrm{m}^{2}\right)$ of Bowman's capsule,
Bowman's space, and glomerular tuft were measured in the digitalized PAS-stained slides using ImageJ software. All available glomeruli (5-11 glomeruli per slide) in each zebrafish were included in the measurements.

\section{Transmission electron microscopy (TEM)}

Zebrafish kidneys were harvested and fixed in EM fixation buffer (1.5\% GA/1\% PF) for $24 \mathrm{~h}$. Subsequently, the renal tissue was fixed with $2.5 \%$ glutaraldehyde/1.2\% acrolein in fixation buffer $(0.1 \mathrm{~mol} / \mathrm{L}$ cacodylate, $0.1 \mathrm{~mol} / \mathrm{L}$ sucrose, $\mathrm{pH}$ 7.4) and $1 \%$ osmium tetroxide, and embedded in epon resin. Ultrathin sections were stained with uranyl acetate. The images were collected using a JEM-1200 EX transmission electron microscope (JEOL, Tokyo, Japan).

The thickness of the glomerular basement membrane (GBM) of zebrafish was analyzed with ImageJ software. One glomerulus per fish was analyzed. Photos of eight distinct areas $(25,000 \times)$ of the glomerular capillaries from each glomerulus at randomly selected places were taken. The mean thickness of the GBM in each photograph was determined by measuring the thickness of 15 nonoverlapping places of the GBM area. The mean of these 8 distinct areas was taken as the thickness for each glomerulus.

\section{Statistical analyses}

Statistical analysis was performed using SPSS Statistics 25 (IBM, Armonk, NY). Differences between groups were analyzed using Student's $t$-test. Data are presented as the mean \pm S.D. Differences with $P<0.05$ were considered as statistically significant.

\section{Results}

\section{Deficiency of lepb has an effect on body weight, length and blood glucose levels in adult zebrafish}

In this study, we first examined the basic parameters, including body weight, length and blood glucose levels, in control and $l e p b^{-/-}$adult zebrafish in both genders at the age of 1.5 years. The body weight of $l e p b^{-/-}$ zebrafish in both genders was significantly increased compared to their respective controls (Fig. 1A; female: $t_{(8.191)}=4.219, P=0.003$; male: $\left.t_{(16)}=2.396, P=0.029\right)$. Accordingly, the body length of $l e p b^{-/-}$zebrafish was also significantly increased compared to the controls, in both genders (Fig. 1B; female: $t_{(12)}=2.680, P=0.020$; 

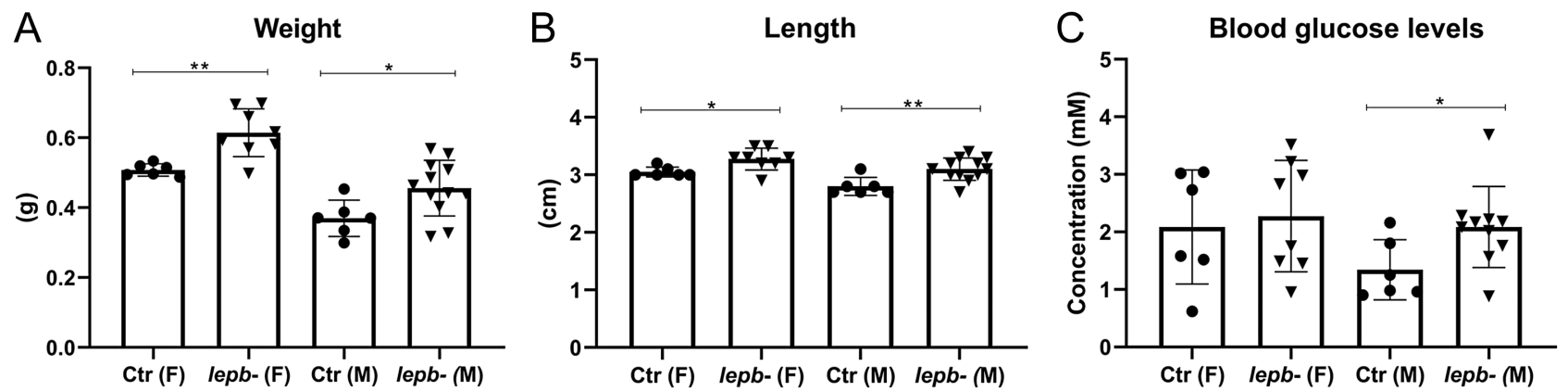

Figure 1

Body weight, length and blood glucose levels in control and lepb-deficient adult zebrafish. (A) The body weight of control and lep $b^{-/-}$female (**P $<0.01$ ) and male ( $\left.{ }^{*} P<0.05\right)$ adult zebrafish. (B) The body length of control and lep $b^{-/-}$female $(* P<0.05)$ and male ( $\left.* * P<0.01\right)$ adult zebrafish. (C) Two hours postprandial blood glucose levels in control and lep $b^{-1-}$ female and male $\left({ }^{\star} P<0.05\right)$ adult zebrafish. Ctr, control zebrafish; lepb-, lepb ${ }^{-1-}$ zebrafish; F, female; M, male.

male: $\left.t_{(16)}=3.266, P=0.005\right)$. Two hours postprandial blood glucose levels (Fig. 1C; female: $t_{(12)}=0.357$, $P=0.727$; male: $\left.t_{(14)}=2.230, P=0.043\right)$ and fasting blood glucose levels (Supplementary Fig. 4; female: $t_{(4)}=0.999$, $P=0.374$; male: $\left.t_{(4)}=3.289, P=0.030\right)$ in $l e p b^{-/-}$male zebrafish group were significantly higher compared to control male zebrafish group, but we did not find that in the female group.

\section{Lepb-deficient adult zebrafish have more visceral fat accumulation}

After observing the obese phenotype in lepb-/- zebrafish, we examined the body fat distribution in zebrafish with a non-invasive MRI approach. Successive slices were imaged from top to bottom in the coronal plane of control and lepb $b^{-1-}$ zebrafish in both genders (Fig. 2). We observed a substantial increase in visceral fat accumulation in both $l e p b^{-/-}$female (Fig. 2B) and male (Fig. 2D) zebrafish compared to control female (Fig. 2A) and male (Fig. 2C) zebrafish. Furthermore, we performed the chemical shift selective (CHESS) imaging to acquire clear fat distribution images (Fig. 2A', B', C' and $\mathrm{D}^{\prime}$ ). A quantitative analysis of body fat from CHESS images clearly showed a significant increase in fat accumulation in $l e p b^{-/-}$zebrafish in both genders compared to their respective controls (Fig. 2E; female: $t_{(10)}=7.057, P<0.001$; male: $\left.t_{(10)}=5.231, P<0.001\right)$.

\section{Lepb-deficient male adult zebrafish develop glomerular hypertrophy}

Subsequently, we investigated the histopathology of the zebrafish kidney, an organ with a high vulnerability under the diabetic condition. We found a substantial increase in the surface area of the glomeruli of lepb ${ }^{-/-}$male zebrafish (Fig. 3B), compared to control male zebrafish (Fig. 3A), on PAS-stained slides. A quantitative analysis of the surface area of the glomeruli showed significant enlargement of Bowman's capsule (Fig. 3C; $t_{(13)}=3.725$, $P=0.003$ ) and glomerular tuft (Fig. 3D; $t_{(13)}=3.176$, $P=0.007)$ in $l e p b^{-/-}$male zebrafish compared to control male zebrafish, indicating that $l e p b^{-/-}$male zebrafish develop glomerular hypertrophy. The surface area of Bowman's space was also larger in $l e p b^{-/}$male zebrafish compared to controls. However, this difference did not reach statistical significance (Fig. 3E; $t_{(13)}=2.108$, $P=0.055)$. Besides, we did not observe severe mesangial matrix expansion in the glomeruli of $l e p b^{-/-}$male zebrafish. Tubular injury and interstitial fibrosis, the final events of chronic tubular injury, contribute to the loss of kidney function. The tubular histology of $l e p b^{-/}$male zebrafish was as normal as control male zebrafish on both PAS- (Fig. 3A and B) and HE-stained slides (Fig. 3F and G), and no signs of the tubular atrophy and interstitial fibrosis were observed.

\section{Lepb-deficient male adult zebrafish show a thickening of the GBM}

Thickening of the GBM is an early sign of diabetic nephropathy (DN). We found a significant increase (2.6 times) in the thickness of GBM in lepb-/- male zebrafish (Fig. 4A, B, C, D and H; $t_{(4)}=10.281, P<0.001$ ) compared to controls via TEM approach. Furthermore, we observed some fibers surrounding the GBM in the glomeruli of lepb-/- male zebrafish (Fig. 4G), suggesting there might have the extracellular matrix accumulation. However, we did not observe obvious podocyte foot process effacement and endothelial cell damage in the glomeruli of $l e p b^{-/-}$ male zebrafish. Besides, the ultrastructure of the renal 


\begin{tabular}{l|l|l|r|r|} 
Journal of & $\mathrm{J}$ He, Y Ding et al. & Leptin deficiency in zebrafish & $\mathbf{2 4 9 : 2}$ & $\mathbf{1 3 0}$ \\
Endocrinology & &
\end{tabular}
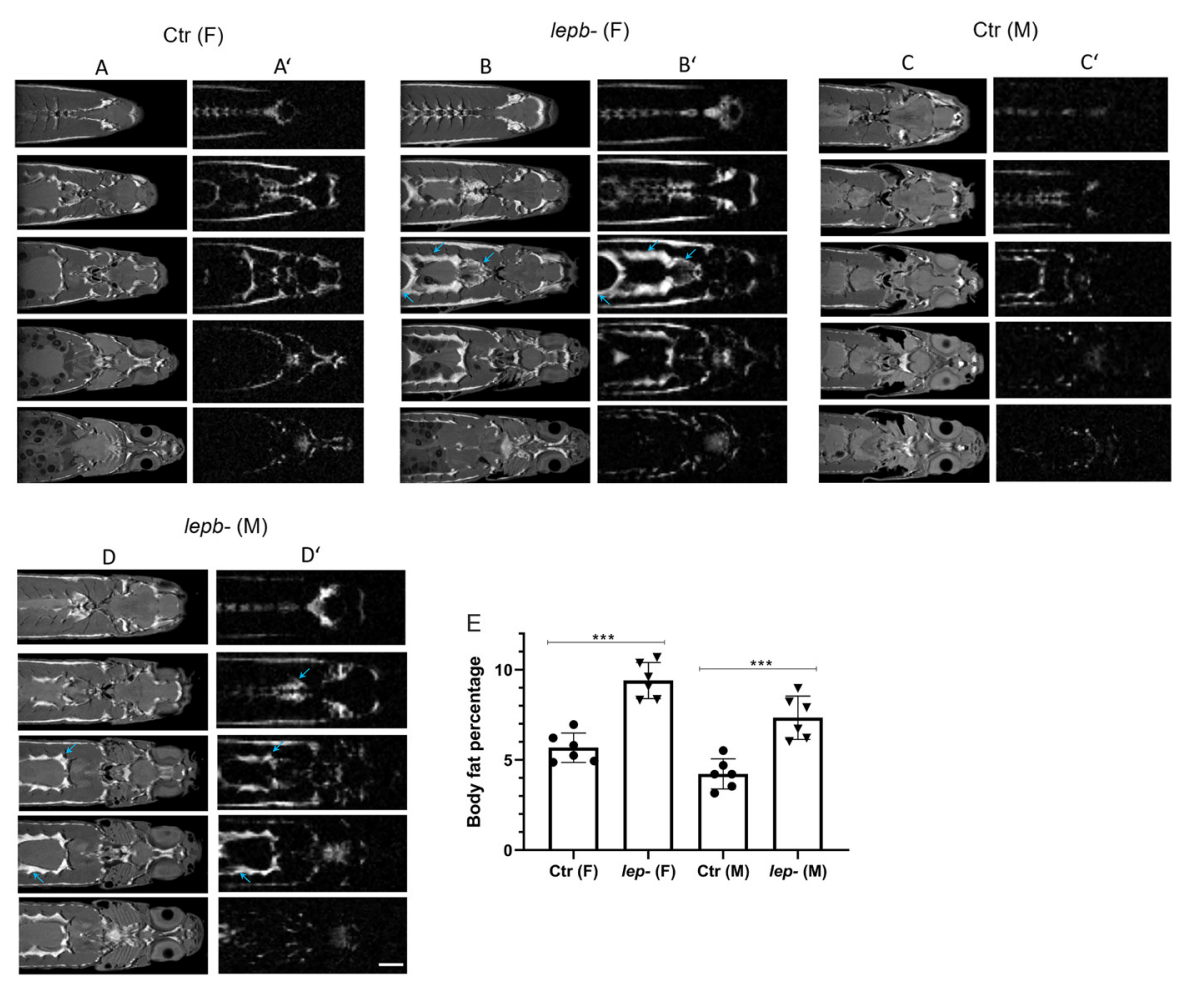

\section{Figure 2}

Magnetic resonance anatomical imaging and selective fat imaging in control and lepb-deficient female and male adult zebrafish. (A, B, C and D) Successive slices (top to bottom) in the coronal plane obtained using T2 weighted RARE pulse sequence. ( $A^{\prime}, B^{\prime}, C^{\prime}$ and $\left.D^{\prime}\right)$ Successive images (top to bottom) of fat distribution in the coronal plane, acquired with Chemical Shift Selective (CHESS) pulse sequence. (A, $A^{\prime}$ ) Control female; (B, B') lep $b^{-/}$ female; (C, C') Control male; ( $\left.D, D^{\prime}\right)$ leph $b^{-1-}$ male adult zebrafish. A substantial visceral fat accumulation was seen in leph $b^{-/-}$adult zebrafish (arrows) as compared to control adult zebrafish (female and male). Scale bar: $2.5 \mathrm{~mm}$. (E) Quantification of body fat in control and lepb-l-female ( $* * \star P<0.001$ ) and male ( $* \star * P<0.001)$ adult zebrafish was measured from CHESS MR images. Ctr, control zebrafish; lepb-, leph-l- zebrafish; F, female; M, male.

proximal tubules of $l e b^{-/-}$male zebrafish was normal and indistinguishable when comparing to control male zebrafish (Fig. 4E and F), which is consistent with the findings from HE and PAS staining.

\section{Discussion}

In the current study, we generated lepb-deficient zebrafish by using the CRISPR/CAS9 gene editing approach. We demonstrated that 1.5 years old lept-/- adult zebrafish in both genders had an increase in body weight, length and visceral fat accumulation compared to their respective controls. Furthermore, we found that the blood glucose levels in $l e p b^{-/}$male adult zebrafish were significantly higher than control male adult zebrafish. Lastly, we showed that $l e p b^{-/-}$male adult zebrafish developed early signs of DN.

There are conflicting reports whether the disruption of leptin signaling induces adiposity in zebrafish. Audira et al. reported that lepa-deficient adult zebrafish display an obese phenotype (Audira et al. 2018). On the other hand,
Michel et al. (2016) did not detect an increased fat mass or higher body weight in lepr-deficient adult zebrafish. In the current study, we observed that all lepb mutants had an increased body weight and body length compared to controls at the adult stage. However, no difference in BMI between control and $l e p b^{-/-}$zebrafish, in both genders, was found (Supplementary Fig. 3C; female: $t_{(12)}=1.120$, $P=0.285$; male: $\left.t_{(16)}=0.070, P=0.945\right)$. It is well known that increased visceral fat accumulation is a typical feature of obesity. In addition, it has been suggested in the literature that body fat percentage and visceral fat level can predict type II diabetes (Lebovitz \& Banerji 2005) or insulin resistance (Kurniawan et al. 2018) better than BMI. To more precisely compare the body fat distribution between control and lepb $b^{-/-}$zebrafish, the successive slices from top to bottom in the coronal plane were imaged via an MRI approach. We found a significantly increased visceral fat accumulation in the lepb-/- zebrafish compared to control zebrafish in both genders. The increase of body weight and visceral fat accumulation in lept-/- zebrafish in both genders jointly suggested that lepb deficiency results in 

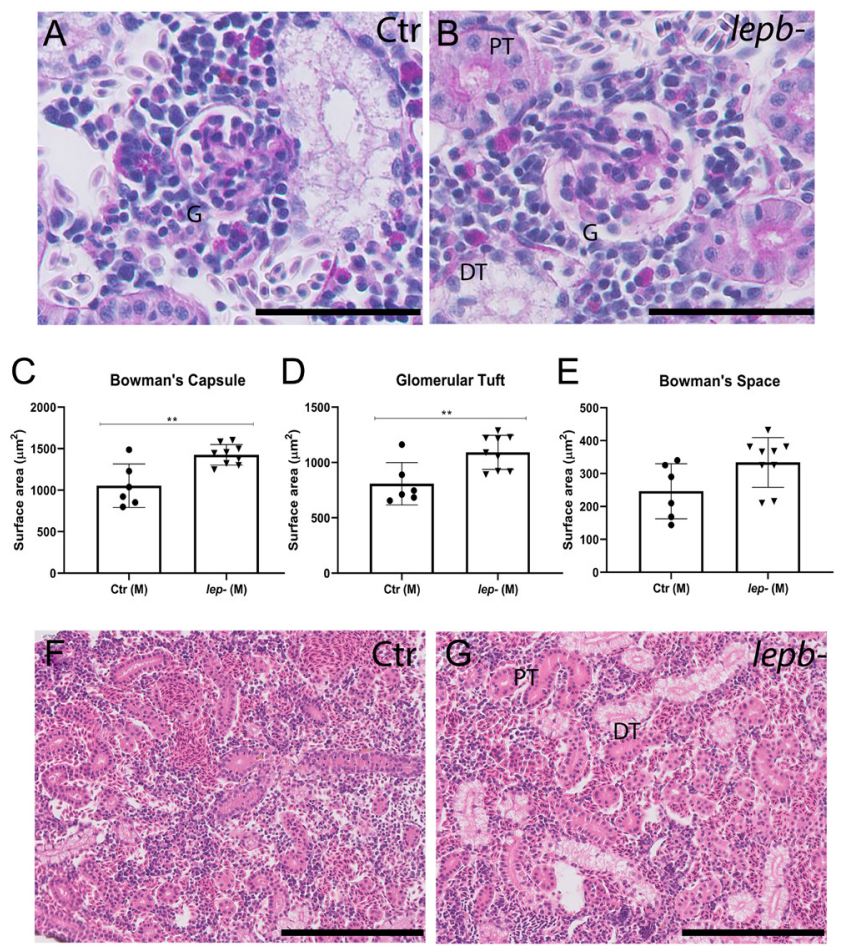

\section{Figure 3}

Lepb-deficient male adult zebrafish develop glomerular hypertrophy. (A and B) Representative images of the glomeruli of control (A) and leph $b^{-l-}$ (B) male adult zebrafish; the scale bars represent $50 \mu \mathrm{m}$; PAS staining. (C) Summary of the surface areas of Bowman's capsule of control and lep $b^{-/-}$male adult zebrafish ( $* * p<0.01$ ). (D) Summary of the surface areas of Glomerular tuft of control and lepb-l- male adult zebrafish ( $* * P<0.01)$. (E) Summary of the surface areas of Bowman's space of control and leph $b^{-/-}$male adult zebrafish. ( $\mathrm{F}$ and G) Representative images of the tubules of control (F) and leph $b^{-/-}(\mathrm{G})$ male adult zebrafish; the scale bars represent $50 \mu \mathrm{m}$; HE staining. Ctr, control zebrafish; lepb-, leph-/- zebrafish; M, male; G, glomeruli; PT, proximal tubule; DT, distal tubule.

adiposity in zebrafish. How can we reconcile our findings with the study of Michel et al. (2016) showing that leprdeficient zebrafish did not develop obesity? The different findings between these two studies might be due to the different age of zebrafish investigated, or different feeding protocols. Alternatively, leptin signaling in zebrafish might also have functions that are independent of the leptin receptor. Besides, to exclude off-target effects in our study, we investigated three different mutants, including $l e p b^{7-17-}, l e p^{8-/ 8-}$ and $l e p b^{7-/ 8-}$ mutants, in the lepb-deficient $\left(l e p b^{-/-}\right)$group. We found that all these three mutants of lep $b^{-/-}$adult zebrafish had an increased body weight and body length, compared to the controls, which makes it unlikely that our results are caused by the off-target effects of the CRISPR-CAS9 procedure.

Recently, researchers have generated different diabetic models in zebrafish. For instance, Zhang et al. developed a zebrafish model for T2DM by overfeeding 4 6 months old fish with diet-induced obesity (DIO) food over 8 weeks, and they showed that these obese fish have increased fasting blood glucose levels (Zang et al. 2017). Olsen et al. induced a type 1 diabetes mellitus (T1DM) in 4 6 months old zebrafish by injecting streptozotocin (STZ) to ablate beta cells from the pancreas (Olsen et al. 2010). This STZinduced T1DM zebrafish model represents several diabetic complications, such as retinal thinning (an early sign of retinopathy) and GBM thickening (an early sign of nephropathy). However, to further investigate diabetes in zebrafish, there is still a lack of a congenital diabetic zebrafish line which does not require time-consuming feeding schemes or invasive procedures which might cause side effects when inducing the diabetic symptoms.

Diabetes is characterised by hyperglycemia which plays a crucial role in the development of diabetes complications. In the current study, we found that 2 hours postprandial blood glucose levels and fasting blood glucose levels of lepb $b^{-/-}$male zebrafish were significantly higher than the levels of control male zebrafish. However, we did not detect this difference in female zebrafish. A review from Wang et al. illustrated that diabetic manifestations in some of the rodent models do not develop in females or are not as apparent as in males (Wang et al. 2014). Therefore, it is very interesting that we got similar results in the current study in a fish model. The increased body weight, length and visceral fat accumulation and higher blood glucose levels in lep $b^{-/-}$male zebrafish collectively indicate that $l e p b$-deficient male adult zebrafish display the features of T2DM. To further explore the glucose homeostasis in the lepb-deficient zebrafish model, it would be very interesting to investigate whether the whole-body glucose level has already increased in the lepb-deficient zebrafish at larvae stage.

Growing literature provides evidence that leptin has a glucose-lowering effect. A previous study reported that the infusion of leptin into the brain could normalize the hyperglycemia in $o b / o b$ mice (Kamohara et al. 1997). Insulin has a robust role in the regulation of glucose homeostasis, and insulin resistance is a fundamental aspect of the etiology of T2DM. Several studies have demonstrated that leptin can improve insulin sensitivity (Shimomura et al. 1999, German et al. 2009). An experimental study from Morton et al. suggested that reduced leptin in the mediobasal hypothalamus leads to severe insulin resistance and glucose intolerance in Koletsky rats, and phosphatidylinositol-3-OH kinase signaling is a vital mediator of this effect (Morton et al. 2005). In addition, Yu et al. reported that leptin could reverse the hyperglycemia 

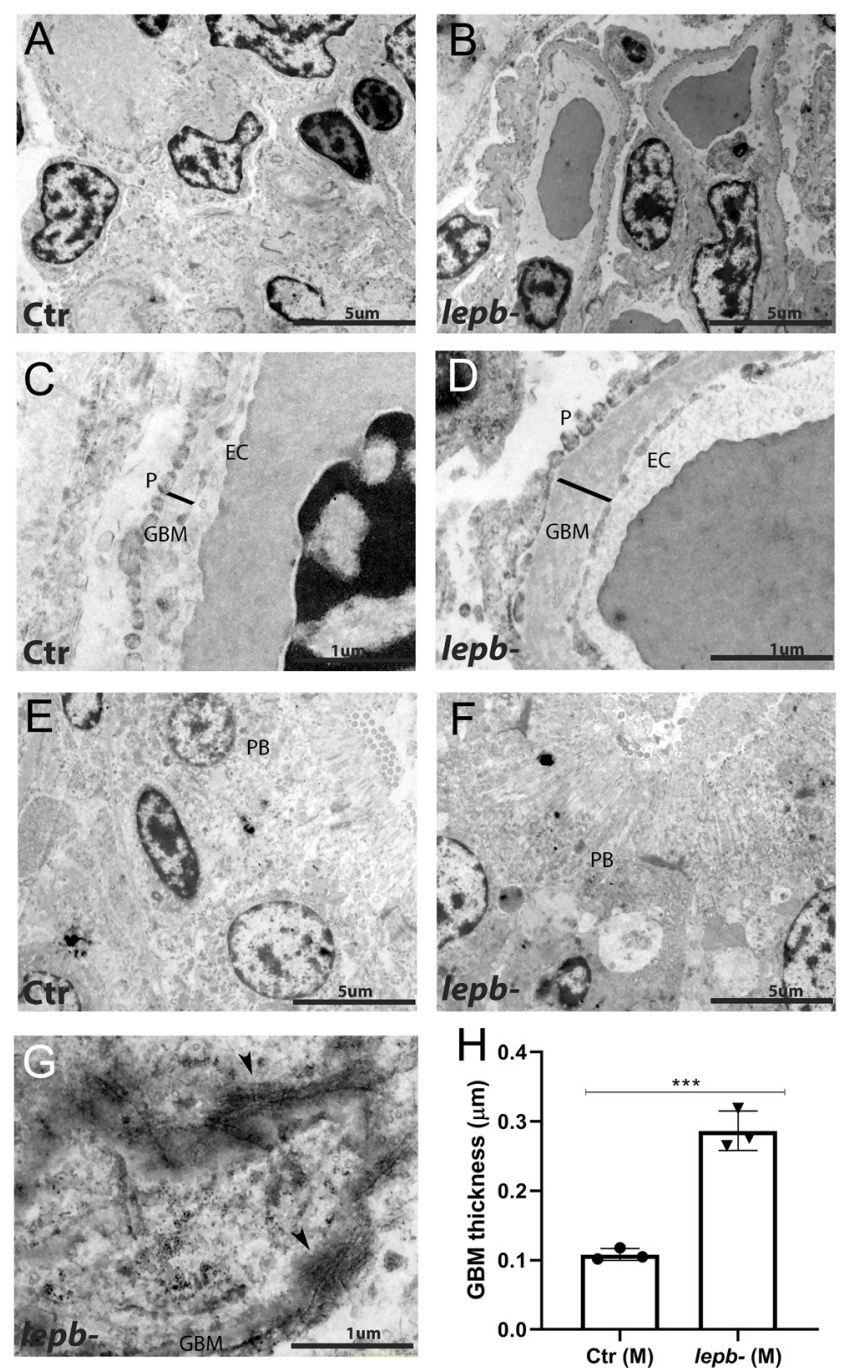

Figure 4

Transmission electron microscopy pictures of glomeruli and tubules from control and lepb-deficient male adult zebrafish. (A and B) Representative images of glomeruli from control (A) and leph-1- (B) male adult zebrafish; magnification 5000×. (C and D) High magnification view of one segment of a glomerular capillary from control (C) and lepb-/- (D) male adult zebrafish (the thickness of GBM was marked by black lines); magnification 25,000×. ( $E$ and $F$ ) Representative images of the tubular brash border of the proximal tubule of control (E) and lepb-1- (F) male adult zebrafish; magnification 5000×. (G) High magnification of the fibrillar mesangial matrix in leph $\mathrm{b}^{-/-}$male adult zebrafish (black arrowheads); magnification $50,000 \times$. (H) Summary of the thickness of GBM in the control and

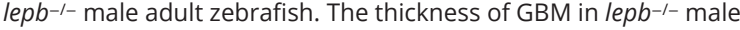
zebrafish is 2.6 times thicker than that in control male zebrafish ( $\star \star \star P<0.001) . C t r$, control zebrafish; lepb-: lep $b^{-1-}$ zebrafish; $\mathrm{M}$, male; GBM, glomerular basement membrane; $\mathrm{P}$, podocytes; EC, endothelial cells; $\mathrm{PB}$, proximal tubular brush border.

and ketosis by suppressing the action of glucagon on the liver and improving the utilization of glucose in the skeletal muscle in insulin-deficient diabetic rodents (Yu et al. 2008). These studies indicated that leptin acts on glucose metabolism in both insulin-dependent and insulin- independent ways. Earlier work from our laboratory had demonstrated that $l e p b$ was significantly downregulated in zebrafish larvae under an insulin-resistance state (MarinJuez et al. 2014). Future studies should investigate whether the diabetic phenotype of lepb mutants is caused by disruption of the insulin signaling pathway.

The segmental anatomy of the nephron is conserved among the vertebrates (McCampbell \& Wingert 2014). Olsen et al. found that STZ-induced T1DM zebrafish have a thickening of the GBM (an early sign of DN) (Olsen et al. 2010). In the current study, we want to address whether $l e p b^{-l-}$ adult zebrafish develop DN or not. We found that the lep $b^{-1-}$ male group had higher blood glucose levels compared to the control male group, but not in the female group. Therefore, the kidney in male zebrafish was further investigated by performing histopathological examination. Glomerular hypertrophy, mesangial expansion, and thickening of the GBM are the particular characteristics of the histopathology of DN (Tervaert et al. 2010). We found that $l e p b^{-/-}$male zebrafish develop glomerular hypertrophy and thickening of the GBM, compared to control male zebrafish. However, we did not observe severe mesangial expansion, podocyte damage, or tubular cell damage in the kidney tissue of lepb-/- male zebrafish. These findings collectively indicate that the renal injury in $l e p b^{-/-}$male zebrafish is relatively mild.

In summary, we demonstrated that lepb regulates glucose homeostasis and adiposity in zebrafish, and deletion of the lepb gene results in the development of T2DM and the early stage of DN. These results suggest that lepb-deficient zebrafish can be used as a T2DM model, and further investigation would give us new insights into the mechanistic function of leptin in T2DM.

\section{Supplementary materials}

This is linked to the online version of the paper at https://doi.org/10.1530/ JOE-20-0437.

\section{Declaration of interest}

The authors declare that there is no conflict of interest that could be perceived as prejudicing the impartiality of the research reported.

\section{Funding}

The work was supported by the Polish National Science Center (grant number 2016/21/N/NZ6/01162).

\section{Author contribution statement}

$\mathrm{J} H$ and $\mathrm{Y} D$ designed the study, analyzed and interpreted the data, and wrote the manuscript. J H, Y D, N N, C J, and M N H E performed experiments 
and the data collection. A A provided technical support and conceptual advice. H J B provided conceptual advice, the experimental design of the study and revisions of the scientific content of the manuscript. H P S conceived the ideas and the experimental design of the study, provided revisions of the scientific content of the manuscript, and supervised the whole study. All authors approved the final version of the manuscript and are fully accountable for all aspects of the study.

\section{Acknowledgements}

The first authors ( $\mathrm{He}$ and Y Ding) were supported by the China Scholarship Council (CSC). We would like to thank Peter Neeskens for the technical support of the transmission electron microscopy (TEM).

\section{References}

Aguilar AJ, Conde-Sieira M, Lopez-Patino MA, Miguez JM \& Soengas JL 2011 In vitro leptin treatment of rainbow trout hypothalamus and hindbrain affects glucosensing and gene expression of neuropeptides involved in food intake regulation. Peptides 32 232-240. (https://doi. org/10.1016/j.peptides.2010.11.007)

Ahima RS, Prabakaran D \& Flier JS 1998 Postnatal leptin surge and regulation of circadian rhythm of leptin by feeding. Implications for energy homeostasis and neuroendocrine function. Journal of Clinical Investigation 101 1020-1027. (https://doi.org/10.1172/JCI1176)

Audira G, Sarasamma S, Chen JR, Juniardi S, Sampurna BP, Liang ST, Lai YH, Lin GM, Hsieh MC \& Hsiao CD 2018 Zebrafish mutants carrying leptin a (lepa) gene deficiency display obesity, anxiety, less aggression and fear, and circadian rhythm and color preference dysregulation. International Journal of Molecular Sciences 194038. (https://doi.org/10.3390/ijms19124038)

Caprio M, Fabbrini E, Isidori AM, Aversa A \& Fabbri A 2001 Leptin in reproduction. Trends in Endocrinology and Metabolism 12 65-72. (https://doi.org/10.1016/s1043-2760(00)00352-0)

Chisada S, Kurokawa T, Murashita K, Ronnestad I, Taniguchi Y, Toyoda A, Sakaki Y, Takeda S \& Yoshiura Y 2014 Leptin receptor-deficient (knockout) medaka, Oryzias latipes, show chronical up-regulated levels of orexigenic neuropeptides, elevated food intake and stage specific effects on growth and fat allocation. General and Comparative Endocrinology 195 9-20. (https://doi.org/10.1016/j. ygcen.2013.10.008)

Denver RJ, Bonett RM \& Boorse GC 2011 Evolution of leptin structure and function. Neuroendocrinology 94 21-38. (https://doi. org/10.1159/000328435)

Drel VR, Mashtalir N, Ilnytska O, Shin J, Li F, Lyzogubov VV \& Obrosova IG 2006 The leptin-deficient (ob/ob) mouse: a new animal model of peripheral neuropathy of type 2 diabetes and obesity. Diabetes 55 3335-3343. (https://doi.org/10.2337/db06-0885)

Farooqi IS, Jebb SA, Langmack G, Lawrence E, Cheetham CH, Prentice AM, Hughes IA, Mccamish MA \& O'Rahilly S 1999 Effects of recombinant leptin therapy in a child with congenital leptin deficiency. New England Journal of Medicine 341 879-884. (https://doi. org/10.1056/NEJM199909163411204)

Fujikawa T, Chuang JC, Sakata I, Ramadori G \& Coppari R 2010 Leptin therapy improves insulin-deficient type 1 diabetes by CNS-dependent mechanisms in mice. PNAS 107 17391-17396. (https://doi. org/10.1073/pnas.1008025107)

Funcke JB, Von Schnurbein J, Lennerz B, Lahr G, Debatin KM, FischerPosovszky P \& Wabitsch M 2014 Monogenic forms of childhood obesity due to mutations in the leptin gene. Molecular and Cellular Pediatrics 1 3. (https://doi.org/10.1186/s40348-014-0003-1)

German J, Kim F, Schwartz GJ, Havel PJ, Rhodes CJ, Schwartz MW \& Morton GJ 2009 Hypothalamic leptin signaling regulates hepatic insulin sensitivity via a neurocircuit involving the vagus nerve. Endocrinology 150 4502-4511. (https://doi.org/10.1210/en.20090445)

German JP, Thaler JP, Wisse BE, Oh-I S, Sarruf DA, Matsen ME, Fischer JD, Taborsky GJ, Schwartz MW \& Morton GJ 2011 Leptin activates a novel CNS mechanism for insulin-independent normalization of severe diabetic hyperglycemia. Endocrinology 152 394-404. (https:// doi.org/10.1210/en.2010-0890)

Gorissen M, Bernier NJ, Nabuurs SB, Flik G \& Huising MO 2009 Two divergent leptin paralogues in zebrafish (Danio rerio) that originate early in teleostean evolution. Journal of Endocrinology 201 329-339. (https://doi.org/10.1677/JOE-09-0034)

Hedbacker K, Birsoy K, Wysocki RW, Asilmaz E, Ahima RS, Farooqi IS \& Friedman JM 2010 Antidiabetic effects of IGFBP2, a leptinregulated gene. Cell Metabolism 11 11-22. (https://doi.org/10.1016/j. cmet.2009.11.007)

Kamohara S, Burcelin R, Halaas JL, Friedman JM \& Charron MJ 1997 Acute stimulation of glucose metabolism in mice by leptin treatment. Nature 389 374-377. (https://doi.org/10.1038/38717)

Kurniawan LB, Bahrun U, Hatta M \& Arif M 2018 Body mass, total body fat percentage, and visceral fat level predict insulin resistance better than waist circumference and body mass index in healthy young male adults in Indonesia. Journal of Clinical Medicine 7 96. (https:// doi.org/10.3390/jcm7050096)

Lebovitz HE \& Banerji MA 2005 Point: visceral adiposity is causally related to insulin resistance. Diabetes Care 28 2322-2325. (https://doi. org/10.2337/diacare.28.9.2322)

Licinio J, Caglayan S, Ozata M, Yildiz BO, De Miranda PB, O'Kirwan F, Whitby R, Liang L, Cohen P, Bhasin S, et al. 2004 Phenotypic effects of leptin replacement on morbid obesity, diabetes mellitus, hypogonadism, and behavior in leptin-deficient adults. PNAS 101 4531-4536. (https://doi.org/10.1073/pnas.0308767101)

Lord GM, Matarese G, Howard JK, Baker RJ, Bloom SR \& Lechler RI 1998 Leptin modulates the T-cell immune response and reverses starvationinduced immunosuppression. Nature 394 897-901. (https://doi. org/10.1038/29795)

Marin-Juez R, Jong-Raadsen S, Yang S \& Spaink HP 2014 Hyperinsulinemia induces insulin resistance and immune suppression via Ptpn6/Shp1 in zebrafish. Journal of Endocrinology 222 229-241. (https://doi.org/10.1530/JOE-14-0178)

Matarese G, Moschos S \& Mantzoros CS 2005 Leptin in immunology. Journal of Immunology 174 3137-3142. (https://doi.org/10.4049/ jimmunol.174.6.3137)

McCampbell KK \& Wingert RA 2014 New tides: using zebrafish to study renal regeneration. Translational Research 163 109-122. (https://doi. org/10.1016/j.trsl.2013.10.003)

Meier U \& Gressner AM 2004 Endocrine regulation of energy metabolism: review of pathobiochemical and clinical chemical aspects of leptin, ghrelin, adiponectin, and resistin. Clinical Chemistry 50 1511-1525. (https://doi.org/10.1373/clinchem.2004.032482)

Michel M, Page-Mccaw PS, Chen W \& Cone RD 2016 Leptin signaling regulates glucose homeostasis, but not adipostasis, in the zebrafish PNAS 113 3084-3089. (https://doi.org/10.1073/pnas.1513212113)

Morton GJ, Gelling RW, Niswender KD, Morrison CD, Rhodes CJ \& Schwartz MW 2005 Leptin regulates insulin sensitivity via phosphatidylinositol-3-OH kinase signaling in mediobasal hypothalamic neurons. Cell Metabolism 2 411-420. (https://doi. org/10.1016/j.cmet.2005.10.009)

Murashita K, Uji S, Yamamoto T, Ronnestad I \& Kurokawa T 2008 Production of recombinant leptin and its effects on food intake in rainbow trout (Oncorhynchus mykiss). Comparative Biochemistry and Physiology: Part B, Biochemistry and Molecular Biology 150 377-384. (https://doi.org/10.1016/j.cbpb.2008.04.007)

Muzzin P, Eisensmith RC, Copeland KC \& Woo SL 1996 Correction of obesity and diabetes in genetically obese mice by leptin gene therapy. PNAS 93 14804-14808. (https://doi.org/10.1073/pnas.93.25.14804) 
Olsen AS, Sarras Jr MP \& Intine RV 2010 Limb regeneration is impaired in an adult zebrafish model of diabetes mellitus. Wound Repair and Regeneration 18 532-542. (https://doi.org/10.1111/j.1524475X.2010.00613.X)

Park S, Hong SM, Sung SR \& Jung HK 2008 Long-term effects of central leptin and resistin on body weight, insulin resistance, and betacell function and mass by the modulation of hypothalamic leptin and insulin signaling. Endocrinology 149 445-454. (https://doi. org/10.1210/en.2007-0754)

Pocai A, Morgan K, Buettner C, Gutierrez-Juarez R, Obici S \& Rossetti L 2005 Central leptin acutely reverses diet-induced hepatic insulin resistance. Diabetes 54 3182-3189. (https://doi.org/10.2337/ diabetes.54.11.3182)

Shimomura I, Hammer RE, Ikemoto S, Brown MS \& Goldstein JL 1999 Leptin reverses insulin resistance and diabetes mellitus in mice with congenital lipodystrophy. Nature 401 73-76. (https://doi. org/10.1038/43448)

Tervaert TW, Mooyaart AL, Amann K, Cohen AH, Cook HT, Drachenberg CB, Ferrario F, Fogo AB, Haas M, De Heer E, et al. 2010 Pathologic classification of diabetic nephropathy. Journal of the
American Society of Nephrology 21 556-563. (https://doi.org/10.1681/ ASN.2010010010)

Volkoff H, Eykelbosh AJ \& Peter RE 2003 Role of leptin in the control of feeding of goldfish Carassius auratus: interactions with cholecystokinin, neuropeptide $\mathrm{Y}$ and orexin $\mathrm{A}$, and modulation by fasting. Brain Research 972 90-109. (https://doi.org/10.1016/s00068993(03)02507-1)

Wang B, Chandrasekera PC \& Pippin JJ 2014 Leptin- and leptin receptordeficient rodent models: relevance for human type 2 diabetes. Current Diabetes Reviews 10 131-145. (https://doi.org/10.2174/157339981066 6140508121012)

Yu X, Park BH, Wang MY, Wang ZV \& Unger RH 2008 Making insulindeficient type 1 diabetic rodents thrive without insulin. PNAS $\mathbf{1 0 5}$ 14070-14075. (https://doi.org/10.1073/pnas.0806993105)

Zang L, Shimada Y \& Nishimura N 2017 Development of a novel zebrafish model for type 2 diabetes mellitus. Scientific Reports 71461. (https://doi.org/10.1038/s41598-017-01432-w)

Zhang Y, Proenca R, Maffei M, Barone M, Leopold L \& Friedman JM 1994 Positional cloning of the mouse obese gene and its human homologue. Nature 372 425-432. (https://doi.org/10.1038/372425a0)

Received in final form 12 February 2021

Accepted 9 March 2021

Accepted Manuscript published online 11 March 2021 (c) 2021 Society for Endocrinology Published by Bioscientifica Ltd. 